\title{
EFECTO DE UN MODIFICADOR ORGÁNICO EN LA GANANCIA DE PESO EN GANADO CEBÚ EN EL TRÓPICO PERUANO
}

\author{
Effects of an Organic Modifier on Body Weight Gain in Zebu Cattle in the \\ Tropics of Peru
}

\author{
Alfredo Delgado C. ${ }^{1,5}$, Antonio Trigueros V. ${ }^{3}$, José Tang P. ${ }^{4}$, Roxana Angelats M. ${ }^{4}$, \\ César Gavidia C. ${ }^{2}$
}

\section{RESUMEN}

\begin{abstract}
El objetivo del presente estudio fue evaluar el efecto de un modificador orgánico (MO) comercial (Modivitasan) sobre la ganancia de peso de ganado cebú. Se utilizaron 36 toretes Nellore de $25 \pm 7.7$ meses de edad y con peso inicial de $208.9 \pm 40.2 \mathrm{~kg}$, distribuidos en un grupo tratado $(\mathrm{n}=20)$ con tres aplicaciones del MO, vía IM, en dosis de $1 \mathrm{ml}$ por cada $50 \mathrm{~kg} / \mathrm{p} . \mathrm{v}$. con intervalos de 30 días, y un grupo control no tratado ( $\mathrm{n}=16)$. Ambos grupos estuvieron en un sistema de pastoreo rotacional en tres potreros sobre una mezcla de Brachiaria decumbens, B. brizantha, Pueraria phaseloides, Paspalum plicatulum y Desmodium ovalifolium por 90 días. Los animales fueron desparasitados con Ivermectina al 3.15\% 15 días previos al inicio del estudio. La ganancia de peso del grupo tratado fue de $43.4 \pm 9.2 \mathrm{~kg}$ y la del grupo control fue de $30.1 \pm 5.4 \mathrm{~kg}(\mathrm{p}<0.01)$. La mayor ganancia de peso en el grupo experimental se explicaría por el aporte adicional de minerales, energía, vitaminas y aminoácidos del modificador orgánico, lo que podría sugerir que el hato se hallaba en una situación de deficiencia marginal importante antes del inicio del experimento. El uso del modificador orgánico constituiría una alternativa para el mejor aprovechamiento de las pasturas de baja calidad del ganado bajo las condiciones de la selva baja del Perú.
\end{abstract}

Palabras clave: toretes, modificador orgánico, tropical, pastoreo

\section{AbSTRACT}

The aim of this study was to evaluate the effects of a commercial organic modifier (Modivitasan) on body weight gain in zebu cattle. Thirty six Nellore steers were used. Animals were $25 \pm 7.7$ months old and had an initial body weight of $208.9 \pm 40.2 \mathrm{~kg}$. They were distributed in a treated group $(\mathrm{n}=20)$ that received three I.M. injections $(1 \mathrm{ml} / 50 \mathrm{~kg}$

\footnotetext{
${ }^{1}$ Clínica de Animales Mayores, ${ }^{2}$ Laboratorio de Medicina Veterinaria Preventiva, Facultad de Medicina Veterinaria, Universidad Nacional Mayor de San Marcos, Lima

${ }^{3}$ Estación Experimental del Trópico, Centro de Investigación IVITA, Universidad Nacional Mayor de San Marcos, Pucallpa

${ }^{4}$ Área de Investigación y Desarrollo, Agrovet Market Animal Health, Lima

${ }^{5}$ E-mail: aldelgadoc@gmail.com
} 
b.w.) of the organic modifier at 30 day-interval, and in a control non-treated group ( $n=16)$. Both groups were kept under a rotational grazing scheme in three paddocks with a mixture of Brachiaria decumbens, B. brizantha, Pueraria phaseloides, Paspalum plicatulum and Desmodium ovalifolium for 90 days. Animals were dewormed with 3.15\% Ivermectin 15 days before the trial. Body weight gain in the treated group was $43.4 \pm 9.2$ $\mathrm{kg}$ and in the control group was $30.1 \pm 5.4 \mathrm{~kg}(\mathrm{p}<0.01)$. The superior body weight gain of the treated group could be explained for the additional supply of minerals, energy, vitamins and proteins contained in the organic modifier in a herd that was under detrimental nutritional conditions before the study. The use of the organic modifier would constitute an alternative for improving the efficient use of low quality pastures in the tropical lowlands of Peru.

Key words: young bull, organic modifier, tropical, pastures

\section{INTRODUCCIÓN}

La selva peruana representa un gran potencial para el desarrollo de la ganadería debido a la existencia de grandes áreas que podrían ser aprovechadas para la explotación pecuaria (Espinoza, 2004). Sin embargo, existen una serie de factores que aún repercuten en la baja productividad del ganado bajo condiciones de trópico, siendo uno de los más importantes la baja calidad nutricional de los recursos forrajeros, especialmente durante la época seca (Araujo-Febres y Rodríguez, 2001).

La alimentación del ganado en selva baja se caracteriza por el uso de pasturas naturales bajo condiciones extensivas; sin embargo, el aporte nutricional de estas no es suficiente para lograr una velocidad de crecimiento y engorde en los animales que permita obtener una saca controlada y sostenible que contribuya a la rentabilidad de los ganaderos (Espinoza, 2004). Adicionalmente, el proceso productivo del rumiante depende en gran medida del consumo voluntario del forraje y su digestibilidad (Obispo et al., 2001).

El bajo valor nutritivo de las gramíneas tropicales constituye uno de los principales factores limitantes para la intensificación de la producción de carne en la región. El contenido de proteína bruta en las pasturas tro- picales oscila entre 3 y $10 \%$, la digestibilidad de la materia orgánica es inferior al 55\% y el contenido en carbohidratos no estructurales es inferior al $10 \%$ de la materia seca (MS). Así, los altos contenidos de fibra y los bajos valores de proteína y digestibilidad de las gramíneas tropicales limitan el consumo voluntario de los bovinos en pastoreo, aumentan la brecha nutricional y comprometen el nivel de respuesta animal (Peruchena, 1999). Un problema adicional lo constituye la estacionalidad en el crecimiento de las pasturas; así, en el trópico peruano, se presenta una estación lluviosa (octubre-abril) y otra de relativa escasez de precipitaciones (mayo-setiembre), que influencian el crecimiento de las pasturas y su valor nutricional (Valdivia et al., 1974).

La constante búsqueda por maximizar el rendimiento del ganado conlleva a un incremento en los requerimientos nutricionales a fin de cubrir la producción adicional de kilogramos de carne. La energía y la proteína son los factores primarios a tener en cuenta; no obstante, su aporte se hace ineficiente si no se tiene en cuenta su interacción con los minerales y las vitaminas, como nutrientes esenciales en la alimentación animal (Repetto et al., 2004).

La búsqueda de alternativas viables orientadas al mejoramiento de los parámetros productivos es necesaria e importante. En la actualidad, los antibióticos, hormonas y otras 
drogas han sido introducidos en el mercado para su uso en la alimentación de los animales domésticos, dado que se afirma que estos productos estimulan el crecimiento o mejoran la salud y el rendimiento de los animales (Espinoza, 2004). Sin embargo, el uso de dichas alternativas, en muchos casos, conlleva a altos costos de producción que no permiten una rentabilidad apropiada. En tal sentido, una suplementación adecuada y económica constituye una herramienta indispensable para favorecer la producción de carne en el trópico. La suplementación permite corregir desbalances en las dietas, aumentar la eficiencia de conversión de las pasturas, y de esa forma, mejorar la ganancia de peso, acortando los ciclos de crecimiento y de engorde del bovino (Peruchena, 1998).

El objetivo del presente estudio fue evaluar el efecto de un modificador orgánico (MO) comercial sobre la ganancia de peso en ganado tropical.

\section{MATERIALES Y MéTODOS}

El estudio se realizó en la Estación Experimental del Trópico del Instituto Veterinario de Investigaciones Tropicales y de Altura (IVITA), de la Facultad de Medicina Veterinaria (FMV), Universidad Nacional Mayor de San Marcos (UNMSM), entre los meses de setiembre de 2009 y enero de 2010. El fundo experimental se encuentra ubicado a 59 km de la ciudad de Pucallpa, en la región Ucayali, zona considerada como bosque húmedo tropical. La temperatura promedio mensual máxima y mínima fluctúa alrededor de los 32 y $22^{\circ} \mathrm{C}$, respectivamente, mientras que la precipitación pluvial anual varía entre 1500 a 2250 mm (SENAMHI, 2012). El periodo de estudio correspondió a la época de inicio de lluvias.

Para la determinación del tamaño de muestra se utilizó la fórmula de diferencia de medias, considerando una diferencia de ganancia de peso promedio de $11 \mathrm{~kg}$ y desviación estándar de 9.5 kg, bajo un nivel de con- fianza del 95\% y un poder estadístico del 80\%. El tamaño muestral mínimo fue de 14 animales por grupo experimental, donde cada animal fue considerado una unidad experimental. En base a esto, se seleccionaron 40 toretes Nellore, con una edad promedio de $25 \pm 7.7$ meses y un peso promedio inicial de $208.9 \pm 40.2 \mathrm{~kg}$. Los animales fueron aleatoriamente asignados a dos grupos experimentales (Tratamiento y Control).

El ensayo tuvo una duración de 105 días. Los primeros 15 días correspondieron a la fase de preparación y los siguientes 90 días a la fase experimental. En la primera fase los animales fueron identificados, pesados y dosificados con una ivermectina en vehículo tixotrópico de efecto prolongado al 3.15\% (Bovimec ${ }^{\circledR}$ etiqueta azul). Los 40 animales formaron un solo grupo de pastoreo en un potrero de 4 ha desde la fase de preparación para uniformizar el comportamiento grupal. La pastura del potrero de preparación y los potreros en la fase experimental tuvieron una composición de forraje similar.

La dieta basal durante la fase experimental consistió en pastoreo ad libitum en tres potreros de 5 ha cada uno, con una mezcla de Braquiaria (Brachiaria decumbens y B. brizantha), Pueraria phaseloides (kudzu), Paspalum plicatulum (pasto negro) y Desmodium ovalifolium, sin predominio significativo entre especies forrajeras. Los animales no recibieron suplementación mineral alguna, según el manejo habitual del centro experimental. Los animales permanecieron 11 días en promedio en cada potrero, realizando cambio de potrero según la disponibilidad de pasto y el grado de pisoteo. La carga animal fue de 3.2 animales por hectárea.

Previo al ingreso de los animales a los potreros, se realizó un análisis de la composición química de las pasturas. Se determinó la digestibilidad in vitro de la materia seca (DIVMS) mediante la técnica descrita por Tilley y Terry (1963), el contenido de proteína cruda según los procedimientos del AOAC (1999), y el contenido de calcio y fósforo de 
acuerdo a las técnicas analíticas estandarizadas en el Laboratorio de Bioquímica, Nutrición y Alimentación Animal de la FMVUNMSM (Arbaiza, 1997). La recolección de muestras de la pastura se hizo siguiendo la metodología propuesta por la Red Internacional de Evaluación de Pastos Tropicales RIEPT (Toledo, 1982).

El grupo Tratado recibió tres aplicaciones del MO, vía intramuscular profunda, en dosis de $1 \mathrm{ml}$ por cada $50 \mathrm{~kg}$ p.v., con intervalo de 30 días, siendo la primera aplicación el día 1 de la fase experimental. El grupo Control no recibió aplicación alguna. El MO (Modivitasan, Agrovet Market) ha sido desarrollado en base a una combinación de nueve aminoácidos esenciales (DL-Metionina, LArginina, L-Histidina, L-Leucina, L-Lisina, LTreonina, L-Triptófano, L-Valina, glutamato de sodio), once compuestos minerales (cloruro de sodio, glicerofosfato de sodio, gluconato de calcio, gluconato de cobalto, gluconato de magnesio, gluconato de manganeso, gluconato de zinc, selenito de sodio, yoduro de potasio), ATP y cuatro vitaminas $\left(\mathrm{A}, \mathrm{B}_{12}, \mathrm{D}_{3} \mathrm{y} \mathrm{E}\right)$.

Los animales fueron pesados al inicio y cada 15 días del estudio, mediante una balanza electrónica con precisión de \pm 1 kg. Las pesadas se hicieron a las 09:00. Se calculó la ganancia de peso obtenida por cada grupo experimental y los datos se analizaron con la prueba de "t" Student, con un nivel de significancia de 0.01 , empleando el programa estadístico Stata v. 10.0.

\section{Resultados y Discusión}

La ganancia de peso de los grupos Tratado y Control se muestra en el Cuadro 1. Los animales del grupo Tratado tuvieron una ganancia diaria de peso de $0.482 \mathrm{~kg}$ en tanto que en el grupo Control se tuvo una ganancia diaria de peso de $0.334 \mathrm{~kg}(\mathrm{p}<0.01)$. La diferencia significativa en la ganancia de peso entre ambos grupos experimentales se evidenció a partir de los 90 días del estudio. Cuatro animales del grupo Control fueron retirados por diversas razones durante la fase inicial del estudio.

Los resultados indican una diferencia en la ganancia de peso de $13.3 \mathrm{~kg}$ entre los dos grupos, a favor de los que recibieron el MO $(\mathrm{p}<0.01)$. La mayor ganancia de peso se explicaría por el aporte de minerales, energía, vitaminas y aminoácidos que contiene el MO y que promueven un mejor metabolismo en los animales tratados, lo que podría sugerir que el ganado con el que se trabajó, se encontraba en una situación de deficiencia nutricional marginal importante antes del inicio del experimento, situación que no le permitía desarrollarse adecuadamente. El peso inicial de los animales de ambos grupos fue bastante bajo en comparación con los estándares de la raza, debido principalmente al deficiente estado de los potreros, el exceso de maleza, y la falta de suplementación mineral. Estos factores condicionaron un deficiente desarrollo corporal y una baja condición de carnes de los animales.

Cuadro 1. Efecto de un modificador orgánico (Modivitasan) sobre la ganancia de peso en toretes Nellore en la selva baja peruana

\begin{tabular}{ccccc}
\hline Grupos & $\begin{array}{c}\mathrm{N}^{\circ} \text { de } \\
\text { animales }\end{array}$ & $\begin{array}{c}\text { Peso inicial } \\
(\mathrm{kg})\end{array}$ & $\begin{array}{c}\text { Peso final } \\
(\mathrm{kg})\end{array}$ & $\begin{array}{c}\text { Ganancia de peso } \\
(\mathrm{kg})\end{array}$ \\
\hline Tratado & 20 & $205.1^{\mathrm{a}} \pm 44.9$ & $248.5 \pm 50.4$ & $43.4^{\mathrm{a}} \pm 9.2$ \\
Control & 16 & $218.1^{\mathrm{a}} \pm 37.6$ & $243.9 \pm 37.6$ & $30.1^{\mathrm{b}} \pm 5.4$ \\
\hline
\end{tabular}

${ }^{a, b}$ Superíndices diferentes indican diferencia estadística $(p<0.01)$ 


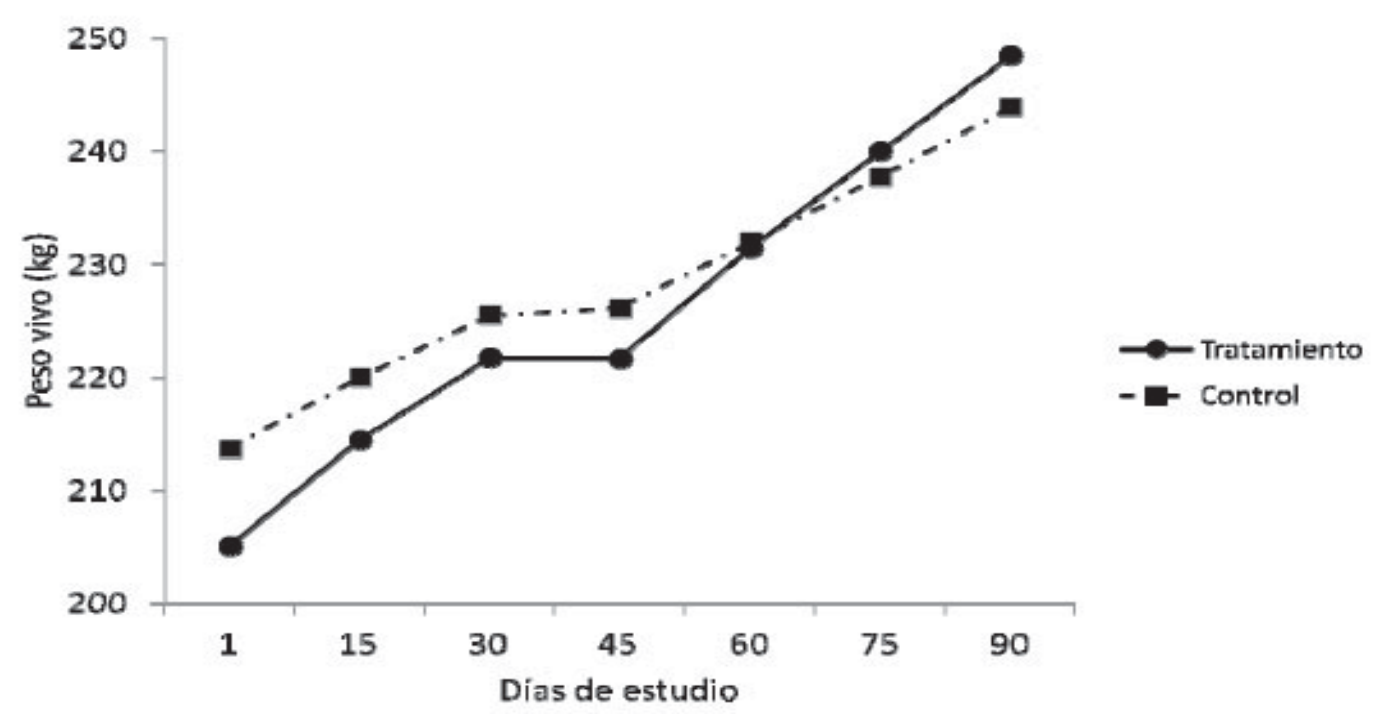

Figura 1. Ganancia de peso del grupo de toretes Nellore que recibió el modificador orgánico (Modivitasan) y del grupo control, no tratado, en la selva baja peruana

Cuadro 2. Análisis proximal porcentual de la pastura

\begin{tabular}{lcccc}
\hline Muestra & DIVMS $^{1}$ & $\begin{array}{c}\text { Proteína } \\
\text { cruda }\end{array}$ & Calcio & Fósforo \\
\hline Brachiaria decumbens $^{2}$ & 56.92 & 7.00 & 0.693 & 0.074 \\
Brachiaria decumbens & 50.57 & 6.55 & 0.583 & 0.113 \\
Brachiaria brizantha & 64.58 & 7.50 & 0.977 & 0.171 \\
Paspalum plicatulum & 47.24 & 6.64 & 0.907 & 0.116 \\
\hline 1 Digestibilidad in vitro de materia seca & \multicolumn{4}{l}{} \\
2 Patrón del Laboratorio de Bioquímica, Nutrición y Alimentación Animal, FMV-UNMSM
\end{tabular}

En otros estudios realizados bajo condiciones similares de pastoreo extensivo en trópico, se obtuvo entre 12 y $18 \%$ de ganancia de peso adicional en el ganado bovino con el uso de anabólicos (Rodríguez, 1989; Durán et al., 2005), resultados menores al obtenido en el presente estudio.

Algunos factores que podrían haber influenciado los resultados, como el efecto del nivel de parasitismo gastrointestinal y la calidad de las pasturas fueron debidamente controlados por el diseño del estudio, dado que los animales fueron sometidos a un tratamiento antiparasitario al inicio del estudio y recibieron el mismo tipo de alimento al pastorear en forma conjunta los potreros asignados en la fase inicial y el periodo experimental. Sin duda, ha existido un crecimiento compensatorio en todos los animales, por efecto de la desparasitación y el ajuste de la carga animal, aunque sin llegar a alcanzar ganancias de peso que podrían esperarse en la zona con el uso de pasturas mejoradas (Morales et al., 1976). La ganancia de peso estuvo más cerca de lo que podría esperarse en explotacio- 
nes de novillos en pasturas tropicales naturales en otras realidades (Rosemberg, 2000).

El resultado del análisis químico de la pastura se muestra en el Cuadro 2. La evidente baja calidad del forraje puede ser uno de los factores que potenció el efecto positivo del MO, dado que pudo haber ocasionado un aumento del consumo voluntario debido a su aporte energético, vitamínico y proteico (Allison, 1985; Del Curto et al., 1990).

Finalmente, la mayor ganancia de peso tuvo un efecto positivo sobre la economía del criador. El costo del tratamiento completo con el MO equivale a S/. 3.00 por animal y si se considera la diferencia en ganancia de peso obtenida y el precio actual de venta de ganado (S/. 4.00 por kilogramo de peso vivo), se obtendría una ganancia neta de S/. 44.20 por animal, en el caso que el ganadero decidiera vender los animales al fin del periodo de trabajo.

\section{Conclusiones}

El uso del modificador orgánico como suplemento nutricional permite obtener un mayor aumento de peso en ganado Nellore de dos años criados bajo las deficitarias condiciones de las pasturas en la selva baja del Perú.

\section{Agradecimientos}

Los autores agradecen al personal de la Estación Experimental del Trópico del Instituto Veterinario de Investigaciones Tropicales y de Altura (IVITA), FMV-UNMSM, destacando el apoyo de los ingenieros César Reyes y Roberto Del Águila, por su asesoría en la realización del presente trabajo.

\section{Literatura Citada}

1. Allison CD. 1985. Factors affecting forage intake by range ruminants: a review. J Range Manage 12(3): 305-311.
2. AOAC. 1999. Official Methods of Analysis of the Association of Official Agricultural Chemists, $16^{\text {th }}$ ed. Arlington, VA, USA: AOAC International. 957 p.

3. Araujo-Febres O, Rodríguez N. 2001. La amonificación de henos como técnica para mejorar su aprovechamiento. Rev Inv Vet, Perú Supl 1: 88-91.

4. Arbaiza T. 1997. Manual de procedimientos para el análisis proximal de alimentos. Lima; Facultad de Medicina Veterinaria, UNMSM. 13 p.

5. DelCurto T, Cochran RC, Harmon DL, Beharka AA, Jacques KA, Towne G, Vanzant ES. 1990. Supplementation of dormant tallgrass-prairie forage: I. Influence varying supplemental protein and (or) energy levels on forage utilization characteristics of beef steers in confinement. J Anim Sci 68: 515-531.

6. Durán E, Calvo C, Díaz R, Sánchez V. 2005. Rentabilidad comparativa entre el pastoreo tradicional y el pastoreo intensivo tecnificado en el trópico seco de Oaxaca. UABJO-EMVZ. [Internet]. Disponible en: http:// www.infolizer.com/1 a mmv 5 eb 2a1n5et/Rentabilidad-comparativa-entre-el-pastoreo-tradicional-y-el-.html

7. Espinoza E. 2004. Efecto comparativo del fósforo asociado a vitaminas (Hematofos B12, complejo B) en el incremento de peso de ganado vacuno mejorado en Iquitos. Tesis de Ingeniero Agrónomo. Iquitos: Univ Nacional de la Amazonía Peruana. 85 p.

8. Morales V, Riesco A, García M, Echevarría M, Santhirasegaram $K$. 1976. Efecto de la carga animal sobre la ganancia de peso de toretes Nellore sobre pasturas mejoradas y sin mejorar. En: V Reunión Nac Asoc Especialistas e Invest Forrajeros del Perú. Huancayo. p 134-135.

9. Obispo N, Pares P, Hidalgo C, Palma J, Godoy S. 2001. Consumo de forraje y ganancia diaria de peso en bovinos de carne en crecimiento suplementados con fuentes proteicas. Zootecnia Trop 19(3): 433-442. 
10. Peruchena CO. 1998. Dietas para la nutrición de bovinos en crecimiento y engorde en el sub-trópico. Publicación Técnica $\mathrm{N}^{\circ}$ 13. Argentina: INTA Corrientes. $24 \mathrm{p}$.

11. Peruchena CO. 1999. Suplementación de bovinos en sistemas pastoriles. En: Publicación Técnica INTA, Jornadas Ganaderas del NEA. Argentina: INTA. p 15-21.

12. Repetto J, Donovan A, García F. 2004. Carencias minerales, limitantes de la producción. Sitio Argentino de Producción Animal [Internet], [05 mayo 2011]. Disponible en: http://www.produccionanimal.com.ar/suplementacion_mineral/ 18-carencias_limitantes_produccion.pdf

13. Rodríguez SLM. 1989. Estudio de la eficiencia del acetato de trembolona 17B estradiol-lactosa, acetato de trembolona 17 B-estradiol-colesterol, benzoato de estradiol más progesterona y zeranol sobre la ganancia de peso en novillo en pastoreo con complementación. Tesis de licenciatura. México DF: Fac de Medicina Veterinaria y Zootecnia, UNAM. 89 p.

14. Rosemberg M. 2000. Utilización del recurso forrajero para el engorde de ganado vacuno. En: Rosemberg M (ed). Producción de ganado vacuno de carne y de doble propósito. Lima: CONCYTEC. p 181-197.

15. SENAMHI. 2012. Servicio Nacional de Meteorología e Hidrología del Perú. [Internet], [12 marzo 2012]. Disponible en: http://www.senamhi.gob.pe/

16. Tilley JMA, Terry RA. 1963. A twostage technique for the in vitro digestion of forage crops. J Br Grassl Soc 18: 104-111.

17. Valdivia $R$, Del Valle O, Echevarría M. 1974. Línea de nutrición animal. Bol Div IVITA-UNMSM 15: 19-26. 